\title{
Binary linear forms over finite sets of integers
}

\author{
by \\ Melvyn B. Nathanson (Bronx, NY), \\ Kevin O'Bryant (Staten Island, NY), \\ Brooke Orosz (New York, NY), ImRe Ruzsa (Budapest), \\ and Manuel Silva (Lisboa)
}

1. Polynomials over finite sets of integers. Let $f=f\left(x_{1}, \ldots, x_{n}\right)$ be a polynomial with integral coefficients. Let $A$ be a nonempty finite set of integers or of congruence classes modulo $m$. We denote by $f(A)$ the image of the function $f$ with domain $A$, that is,

$$
f(A)=\left\{f\left(a_{1}, \ldots, a_{n}\right): a_{i} \in A \text { for } i=1, \ldots, n\right\} .
$$

If $f$ is a polynomial in $n$ variables, then $|f(A)| \leq|A|^{n}$.

The classical examples are the polynomials $x_{1}+x_{2}$ and $x_{1}-x_{2}$. The sumset $A+A$ is the set $s(A)$ for the polynomial $s\left(x_{1}, x_{2}\right)=x_{1}+x_{2}$, and the difference set $A-A$ is the set $d(A)$ for the polynomial $d\left(x_{1}, x_{2}\right)=x_{1}-x_{2}$. For any arithmetic progression $A$ or, more generally, any symmetric set $A$ of integers, we have $|d(A)|=|s(A)|$, but for "most" sets $A$ the difference set contains more elements than the sumset. It had been conjectured (cf. Croft [1], Marica [3], Nathanson [7, 6]) that $|d(A)| \geq|s(A)|$ for every set $A$, but the set $A=\{0,2,3,4,7,11,12,14\}$ is a counterexample, since

$$
|A-A|=|d(A)|=25
$$

and

$$
|A+A|=|s(A)|=26 .
$$

Sets with more sums than differences have also been studied by Hegarty [2] and Martin and O'Bryant [4]. This suggests the following problem.

2000 Mathematics Subject Classification: Primary 11B75, 11D04, 11P99, 05B10.

Key words and phrases: additive number theory, combinatorial number theory, sumsets, difference sets.

The work of M.B.N. was supported in part by grants from the NSA Mathematical Sciences Program and the PSC-CUNY Research Award Program. 
Problem 1. Let $f\left(x_{1}, \ldots, x_{n}\right)$ and $g\left(x_{1}, \ldots, x_{n}\right)$ be polynomials with integer coefficients. Determine if there exist finite sets $A, B, C$ of positive integers with $|C|>1$ such that

$$
\left\{\begin{array}{l}
|f(A)|>|g(A)|, \\
|f(B)|<|g(B)|, \\
|f(C)|=|g(C)| .
\end{array}\right.
$$

There is a stronger form of Problem 1.

Problem 2. Let $f\left(x_{1}, \ldots, x_{n}\right)$ and $g\left(x_{1}, \ldots, x_{n}\right)$ be polynomials with integer coefficients. Does there exist a sequence $\left\{A_{i}\right\}_{i=1}^{\infty}$ of finite sets of integers such that

$$
\lim _{i \rightarrow \infty} \frac{\left|f\left(A_{i}\right)\right|}{\left|g\left(A_{i}\right)\right|}=\infty ?
$$

Does there exist a sequence $\left\{C_{i}\right\}_{i=1}^{\infty}$ of finite sets of integers with $\lim _{i \rightarrow \infty}\left|C_{i}\right|$ $=\infty$ and $\left|f\left(C_{i}\right)\right|=\left|g\left(C_{i}\right)\right|$ for all $i$ ?

Linear polynomials constitute an important special case.

Problem 3. Let $f\left(x_{1}, \ldots, x_{n}\right)=u_{1} x_{1}+\cdots+u_{n} x_{n}$ and $g\left(x_{1}, \ldots, x_{n}\right)=$ $v_{1} x_{1}+\cdots+v_{n} x_{n}$ be linear forms with integer coefficients. Do there exist finite sets $A, B, C$ of integers with $|C|>1$ that satisfy (1)?

The interval of integers $[a, b]$ is the set of integers $\{a, a+1, \ldots, b\}$. For any integer $u$ and sets $A$ and $B$ of integers, we define the dilation

$$
u * A=\{u a: a \in A\}
$$

and the sumset

$$
A+B=\{a+b: a \in A \text { and } b \in B\} .
$$

If $f\left(x_{1}, \ldots, x_{n}\right)=u_{1} x_{1}+\cdots+u_{n} x_{n}$ is a linear form, then

$$
f(A)=u_{1} * A+\cdots+u_{n} * A .
$$

Sets of integers $A$ and $B$ are affinely equivalent if there are rational numbers $u \neq 0$ and $v$ such that $B=u * A+\{v\}$. In this case,

$$
f(B)=f(u * A+\{v\})=u * f(A)+\{f(v, \ldots, v)\},
$$

hence

$$
|f(A)|=|f(B)| .
$$

Note that every two-element set is affinely equivalent to the set $\{0,1\}$, and that every set $A$ of integers with $1<|A|<\infty$ is affinely equivalent to a set $A^{\prime}$ such that $0 \in A^{\prime}$ and $A^{\prime} \backslash\{0\}$ is a set of relatively prime positive integers. The following theorem implies that Problem 3 is equivalent to Problem 2 in the case of linear forms. 
THEOREM 1. Let $f\left(x_{1}, \ldots, x_{n}\right)$ and $g\left(x_{1}, \ldots, x_{n}\right)$ be linear forms with integer coefficients, and let $A$ and $C$ be finite sets of integers such that $|f(A)|>|g(A)|,|f(C)|=|g(C)|$, and $|C|>1$. There exist sequences $\left\{A_{i}\right\}_{i=1}^{\infty}$ and $\left\{C_{i}\right\}_{i=1}^{\infty}$ of finite sets of integers with $\lim _{i \rightarrow \infty}\left|C_{i}\right|=\infty$ such that

$$
\lim _{i \rightarrow \infty} \frac{\left|f\left(A_{i}\right)\right|}{\left|g\left(A_{i}\right)\right|}=\infty
$$

and $\left|f\left(C_{i}\right)\right|=\left|g\left(C_{i}\right)\right|$ for all $i$.

Proof. Let $f\left(x_{1}, \ldots, x_{n}\right)=\sum_{i=1}^{n} u_{i} x_{i}$ and $g\left(x_{1}, \ldots, x_{n}\right)=\sum_{i=1}^{n} v_{i} x_{i}$ be linear forms with integer coefficients, and let $A$ be a finite set of integers. We define

$$
m_{f, g}(A)=\max (|s|: s \in A \cup f(A) \cup g(A)),
$$

and choose an integer

$$
M>2 m_{f, g}(A) .
$$

Let

$$
A_{M}=A+M * A=\left\{a+M a^{\prime}: a, a^{\prime} \in A\right\} .
$$

If $a_{1}, a_{1}^{\prime}, a_{2}, a_{2}^{\prime} \in A$ and $a_{1}+M a_{1}^{\prime}=a_{2}+M a_{2}^{\prime}$, then $a_{1}-a_{2}=M\left(a_{2}^{\prime}-a_{1}^{\prime}\right)$. Since

$$
M\left|a_{2}^{\prime}-a_{1}^{\prime}\right|=\left|a_{1}-a_{2}\right| \leq\left|a_{1}\right|+\left|a_{2}\right| \leq 2 m_{f, g}(A)<M,
$$

it follows that $a_{1}^{\prime}=a_{2}^{\prime}$ and so $a_{1}=a_{2}$ and

$$
\left|A_{M}\right|=|A|^{2} \text {. }
$$

The identity

$$
\sum_{i=1}^{n} u_{i}\left(a_{i}+M a_{i}^{\prime}\right)=\sum_{i=1}^{n} u_{i} a_{i}+M \sum_{i=1}^{n} u_{i} a_{i}^{\prime}
$$

implies that

$$
f\left(A_{M}\right)=f(A)+M * f(A) .
$$

If $s_{1}, s_{1}^{\prime}, s_{2}, s_{2}^{\prime} \in f(A)$ and

$$
s_{1}+M s_{1}^{\prime}=s_{2}+M s_{2}^{\prime},
$$

it again follows that $s_{1}=s_{2}, s_{1}^{\prime}=s_{2}^{\prime}$, and

$$
\left|f\left(A_{M}\right)\right|=|f(A)|^{2} .
$$

Similarly,

$$
\left|g\left(A_{M}\right)\right|=|g(A)|^{2}
$$

and so

$$
\frac{\left|f\left(A_{M}\right)\right|}{\left|g\left(A_{M}\right)\right|}=\left(\frac{|f(A)|}{|g(A)|}\right)^{2} .
$$

The theorem follows by iterating this construction. 
In the case of binary linear forms, we write $f(x, y)=u x+v y$ instead of $f\left(x_{1}, x_{2}\right)=u_{1} x_{1}+u_{2} x_{2}$. We are interested only in the cardinality of the image of $f(x, y)$ on a finite set $A$ of integers. We shall always assume that $u v \neq 0$. If $(u, v)=d>1$ and $g(x, y)=(u / d) x+(v / d) y$, then $|f(A)|=|g(A)|$. Thus, we can assume that $(u, v)=1$. Similarly, if $h(x, y)=v x+u y$, then $|f(A)|=|h(A)|$, and so we can assume that $|u| \geq|v|$. Finally, if $\ell(x, y)=$ $-u x-v y$, then $|f(A)|=|\ell(A)|$, and we can assume that $u>0$. Therefore, it suffices to consider only binary linear forms $f(x, y)=u x+v y$ that have been normalized so that

$$
u \geq|v| \geq 1 \quad \text { and } \quad(u, v)=1 .
$$

Problem 4. Let $f(x, y)=u_{1} x+v_{1} y$ and $g(x, y)=u_{2} x+v_{2} y$ be normalized binary linear forms with nonzero integer coefficients $\left(u_{1}, v_{1}\right) \neq\left(u_{2}, v_{2}\right)$. Do there exist finite sets of integers $A$ and $B$ such that $|f(A)|>|g(A)|$ and $|f(B)|<|g(B)|$ ?

In this paper we shall prove that the answer to the question in Problem 4 is "yes".

2. Pairs of binary linear forms with $u_{1}, u_{2} \geq 2$. In this section we prove that if $f(x, y)=u_{1} x+v_{1} y$ and $g(x, y)=u_{2} x+v_{2} y$ are normalized binary linear forms with $u_{1} \geq 2, u_{2} \geq 2$, and $\left(u_{1}, v_{1}\right) \neq\left(u_{2}, v_{2}\right)$, then there exist finite sets $A, B, C$ of integers such that $|f(A)|<|g(A)|,|f(B)|>$ $|g(B)|$, and $|f(C)|=|g(C)|$.

THEOREM 2. For $u>|v| \geq 1$ and $(u, v)=1$, consider the normalized binary linear form

$$
f(x, y)=u x+v y .
$$

(i) If $|A|=2$, then $|f(A)|=4$.

(ii) If $u \geq 3$ and $|A|=3$, then $|f(A)|=8$ or 9 , and $|f(A)|=8$ if and only if $A$ is affinely equivalent to one of the two sets

$$
\{0,|v|, u\} \quad \text { and } \quad\{0,|v|, u+|v|\} .
$$

(iii) If $u=2$ and $|A|=3$, then $|f(A)|<9$ if and only if $A$ is affinely equivalent to one of the two sets

$$
\{0,1,2\} \text { and }\{0,1,3\} \text {. }
$$

Moreover, $|f(\{0,1,2\})|=7$ and $|f(\{0,1,3\})|=8$.

(iv) If $f(x, y)=u x+v y$, and $g(x, y)=u x-v y$, then $|f(A)|=|g(A)|$ for every set $A$ with $|A|=3$.

Proof. If $f(x, y)=u x+v y$ is a normalized binary linear form, then $f(\{0,1\})=\{0, v, u, u+v\}$ and so $|f(\{0,1\})|=4$. Since every set $A$ with $|A|=2$ is affinely equivalent to $\{0,1\}$, it follows that if $|A|=2$, then $|f(A)|=4$. This proves (i). 
Let $|A|=3$. The set $A$ is affinely equivalent to a set $A^{\prime}$ such that $\min \left(A^{\prime}\right)=0$ and $\operatorname{gcd}\left(A^{\prime}\right)=1$. If $|f(A)| \leq 8$, then there exist $x_{1}, y_{1}, x_{2}, y_{2}$ $\in A^{\prime}$ such that

$$
u x_{1}+v y_{1}=u x_{2}+v y_{2} \quad \text { and } \quad\left(x_{1}, y_{1}\right) \neq\left(x_{2}, y_{2}\right) .
$$

It follows from (i) that $\left|\left\{x_{1}, y_{1}, x_{2}, y_{2}\right\}\right|>2$ and so

$$
\left\{x_{1}, y_{1}, x_{2}, y_{2}\right\}=\left\{a_{1}, a_{2}, a_{3}\right\}=A^{\prime} .
$$

There are three possibilities: Either

$$
u a_{1}+v a_{2}=u a_{1}+v a_{3},
$$

or

$$
u a_{1}+v a_{2}=u a_{3}+v a_{1},
$$

or

$$
u a_{1}+v a_{1}=u a_{2}+v a_{3} .
$$

In the first case, $a_{2}=a_{3}$, which is absurd.

In the second case, we have

$$
u\left(a_{1}-a_{3}\right)=v\left(a_{1}-a_{2}\right) .
$$

Since $(u, v)=1$, there exists an integer $r$ such that

$$
a_{1}-a_{2}=r u, \quad a_{1}-a_{3}=r v, \quad a_{3}-a_{2}=r(u-v) .
$$

Since $0 \in A^{\prime}$, it follows that $r$ divides each integer in $A^{\prime}$, and so $r= \pm 1$. If $a_{1}=0$, then $r=-1, a_{2}=u, a_{3}=v=|v|$, and $A^{\prime}=\{0,|v|, u\}$. If $a_{2}=0$, then $r=1, a_{1}=u$, and $a_{3}=u-v$. If $v>0$, then $A^{\prime}=\{0, u-|v|, u\}$. If $v<0$, then $A^{\prime}=\{0, u, u+|v|\}$. If $a_{3}=0$, then $a_{2}=-r(u-v)$ and so $r=-1, a_{1}=-v=|v|, a_{2}=u-v=u+|v|$, and $A^{\prime}=\{0,|v|, u+|v|\}$.

In the third case,

$$
u\left(a_{1}-a_{2}\right)=v\left(a_{3}-a_{1}\right)
$$

and there is an integer $r= \pm 1$ such that

$$
a_{1}-a_{2}=r v, \quad a_{3}-a_{1}=r u, \quad a_{3}-a_{2}=r(u+v) .
$$

If $a_{1}=0$, then $r=1, a_{3}=u, a_{2}=-v=|v|$, and $A^{\prime}=\{0,|v|, u\}$. If $a_{2}=0$, then $r=1, a_{1}=v=|v|, a_{3}=u+v=u+|v|$, and $A^{\prime}=\{0,|v|, u+|v|\}$. If $a_{3}=0$, then $r=-1, a_{1}=u$, and $a_{2}=u+v$. If $v>0$, then $A^{\prime}=\{0, u, u+|v|\}$. If $v<0$, then $A^{\prime}=\{0, u-|v|, u\}$.

Since the two sets $\{0,|v|, u\}$ and $\{0, u-|v|, u\}$ are affinely equivalent, and the two sets $\{0, u, u+|v|\}$ and $\{0,|v|, u+|v|\}$ are also affinely equivalent, it follows that the sets $\{0,|v|, u\}$ and $\{0, u, u+|v|\}$ are, up to affine equivalence, the only possible solutions of $|f(A)| \leq 8$ with $|A|=3$.

We shall prove that if $u \geq 3$, then $|f(A)|=8$ for both these sets. Let $v>0$ and $f(x, y)=u x+v y$. If $A=\{0, v, u\}$, then

$$
f(A)=\left\{0, v^{2}, u v, u^{2}, u v+v^{2}, 2 u v, u^{2}+v^{2}, u v+u^{2}\right\} .
$$


Since $2 u v<u^{2}+v^{2}$ for $v<u$, we have

$$
0<v^{2}<u v<u v+v^{2}<2 u v<u^{2}+v^{2}<u v+u^{2}
$$

and

$$
u v<u^{2}<u^{2}+v^{2}
$$

If $u^{2}=2 u v$, then $u=2 v=2$ since $(u, v)=1$. If $u^{2}=u v+v^{2}$, then $u / v=(1+\sqrt{5}) / 2$, which is impossible since $u / v$ is rational. Therefore, $|f(A)|=8$.

If $v>0$ and $A=\{0, v, u+v\}$, then

$$
f(A)=\left\{0, v^{2}, u v, u v+v^{2}, 2 u v+v^{2}, u^{2}+u v, u^{2}+u v+v^{2}, u^{2}+2 u v+v^{2}\right\} \text {. }
$$

We have

$$
0<v^{2}<u v<u v+v^{2}<2 u v+v^{2}<u^{2}+u v+v^{2}<u^{2}+2 u v+u^{2}
$$

and

$$
u v+v^{2}<u^{2}+v^{2}<u^{2}+u v+v^{2} .
$$

If $u^{2}+v^{2}=2 u v+v^{2}$, then $u=2 v=2$, which is false, and so $|f(A)|=8$. The case $u \geq 3$ and $v<0$ is similar. This proves (ii).

If $u=2$, then $v= \pm 1$ and $f(x, y)=2 x \pm y$. Up to affine equivalence, the sets $A$ with $|f(A)| \leq 8$ are $\{0,1,2\}$ and $\{0,1,3\}$. For these sets, $|f(\{0,1,2\})|=7$ and $|f(\{0,1,3\})|=8$. This proves (iii).

To obtain (iv), we observe that the binary linear forms $f(x, y)=u x+v y$ and $g(x, y)=u x-v y$ generate the same exceptional sets, and so $|f(A)|=$ $|g(A)|$ whenever $|A|=3$. This completes the proof.

LEMMA 1. Let

$$
F(x, y)=c_{0} x^{k}+c_{1} x^{k-1} y+\cdots+c_{k-1} x y^{k-1}+c_{k} y^{k}
$$

be a nonzero homogeneous polynomial with integer coefficients. Let $j$ be the largest integer such that $c_{j} \neq 0$. If $u$ and $v$ are relatively prime nonzero integers such that $F(u, v)=0$, then $\left|c_{j}\right| \geq|u|$.

We call $c_{j}$ the last coefficient in the polynomial $F(x, y)$.

Proof. If

$$
F(u, v)=\sum_{i=0}^{k} c_{i} u^{k-i} v^{i}=u^{k-j} \sum_{i=0}^{j} c_{i} u^{j-i} v^{i}=0
$$

then

$$
u \sum_{i=0}^{j-1} c_{i} u^{j-i-1} v^{i}=-c_{j} v^{j}
$$

and so $u$ divides $c_{j} v^{j}$. Since $(u, v)=1$ and $c_{j} \neq 0$, it follows that $u$ divides $c_{j}$ and so $\left|c_{j}\right| \geq|u|$. 
Theorem 3. Let

$$
f(x, y)=u_{1} x+v_{1} y \quad \text { and } \quad g(x, y)=u_{2} x+v_{2} y
$$

be normalized binary linear forms with

$$
u_{1} \geq 2, \quad u_{2} \geq 2,
$$

and

$$
\left(u_{1},\left|v_{1}\right|\right) \neq\left(u_{2},\left|v_{2}\right|\right) .
$$

There exist sets $A$ and $B$ with $|A|=|B|=3$ such that

$$
|f(A)|<|g(A)| \text { and }|f(B)|>|g(B)| .
$$

Proof. If $u_{1}<u_{2}$ and $u_{2} \neq u_{1}+\left|v_{1}\right|$, then the sets $A=\left\{0,\left|v_{1}\right|, u_{1}\right\}$ and $B=\left\{0,\left|v_{2}\right|, u_{2}\right\}$ satisfy $|f(A)|=|g(B)| \leq 8$ and $|f(B)|=|g(A)|=9$. If $u_{1}<u_{2}$ and $u_{2}=u_{1}+\left|v_{1}\right|$, then $u_{2}+\left|v_{2}\right|>u_{1}+\left|v_{1}\right|$ and the sets $A=\left\{0,\left|v_{1}\right|, u_{1}\right\}$ and $B=\left\{0,\left|v_{2}\right|, u_{2}+\left|v_{2}\right|\right\}$ satisfy $|f(A)|=|g(B)|=8$ and $|f(B)|=|g(A)|=9$.

If $u_{1}=u_{2}$ and $\left|v_{1}\right|<\left|v_{2}\right|$, then sets $A=\left\{0,\left|v_{1}\right|, u_{1}+\left|v_{1}\right|\right\}$ and $B=$ $\left\{0,\left|v_{2}\right|, u_{2}+\left|v_{2}\right|\right\}$ satisfy $|f(A)|=|g(B)|=8$ and $|f(B)|=|g(A)|=9$. This completes the proof.

THEOREM 4. Let

$$
f(x, y)=u x+v y \quad \text { and } \quad g(x, y)=u x-v y
$$

be normalized binary linear forms with $u>v \geq 1$. For $u=2$, if

$$
A=\{0,3,4,6\}, \quad B=\{0,4,6,7\}
$$

then

$$
|f(A)|=13>12=|g(A)|, \quad|f(B)|=13<14=|g(B)| .
$$

For $u \geq 3$, if

$$
A=\left\{0, u^{2}-v^{2}, u^{2}, u^{2}+u v\right\}, \quad B=\left\{0, u^{2}-u v, u^{2}-v^{2}, u^{2}\right\}
$$

then

$$
|f(A)|=14>13=|g(A)|, \quad|f(B)|=13<14=|g(B)| .
$$

Proof. For $u=2$ and sets $A=\{0,3,4,6\}$ and $B=\{0,4,6,7\}$, we have

$$
\begin{aligned}
& f(A)=\{0,3,4,6,8,9,10,11,12,14,15,16,18\}, \\
& g(A)=\{-6,-4,-3,0,2,3,4,5,6,8,9,12\}, \\
& f(B)=\{0,4,6,7,8,12,14,15,16,18,19,20,21\}, \\
& g(B)=\{-7,-6,-4,0,1,2,4,5,6,7,8,10,12,14\}
\end{aligned}
$$

with $|f(A)|=|f(B)|=13,|g(A)|=12$, and $|g(B)|=14$.

Let $u \geq 3$ and $A=\left\{0, u^{2}-v^{2}, u^{2}, u^{2}+u v\right\}$. We list the elements of the set $f(A)=\{u x+v y: x, y \in A\}$ in the following table: 


\begin{tabular}{ccccc}
\hline$f(A)$ & 0 & $u^{2}-v^{2}$ & $u^{2}$ & $u^{2}+u v$ \\
\hline 0 & 0 & $u^{2} v-v^{3}$ & $u^{2} v$ & $u^{2} v+u v^{2}$ \\
$u^{2}-v^{2}$ & $u^{3}-u v^{2}$ & $u^{3}+u^{2} v-u v^{2}-v^{3}$ & $u^{3}+u^{2} v-u v^{2}$ & $u^{3}+u^{2} v$ \\
$u^{2}$ & $u^{3}$ & $u^{3}+u^{2} v-v^{3}$ & $\frac{u^{3}+u^{2} v}{u^{3}+2 u^{2} v}$ & $u^{3}+u^{2} v+u v^{2}$ \\
$u^{2}+u v$ & $u^{3}+u^{2} v$ & $u^{3}+2 u^{2} v-v^{3}$ & $u^{2} v+u v^{2}$ \\
\hline
\end{tabular}

The number $u^{3}+u^{2} v$ occurs three times in this table, and so $|f(A)| \leq 14$. By Lemma 1, if two numbers in the table are equal for positive integers $u$ and $v$ with $u \geq 3$ and $(u, v)=1$, then the difference of the two numbers is an expression of the form $F(u, v)$, where $F(x, y)$ is a homogeneous polynomial of degree 3 with last coefficient at least 3 . Since $1 \leq v<u$, the numbers in the table are increasing from left to right in each row and from top to bottom in each column. The following ten numbers in the set $f(A)$ are strictly increasing:

$$
\begin{aligned}
0 & <u^{2} v-v^{3}<u^{3}-u v^{2}<u^{3}+u^{2} v-u v^{2}-v^{3} \\
& <u^{3}+u^{2} v-u v^{2}<u^{3}+u^{2} v-v^{3}<u^{3}+u^{2} v \\
& <u^{3}+u^{2} v+u v^{2}<u^{3}+2 u^{2} v<u^{3}+2 u^{2}+u v^{2} .
\end{aligned}
$$

The other four numbers in $f(A)$ satisfy

$$
u^{2} v<u^{3}<u^{3}+2 u^{2} v-v^{3}
$$

and

$$
u^{2} v<u^{2} v+u v^{2}<u^{3}+2 u^{2} v-v^{3} .
$$

Comparing numbers among the three chains of inequalities, we see that there is no difference with last coefficient greater than 2, and so $|f(A)|=14$.

Consider now the set $g(A)=\{u x-v y: x, y \in A\}$, whose elements are listed in the following table:

\begin{tabular}{ccccc}
\hline$g(A)$ & 0 & $u^{2}-v^{2}$ & $u^{2}$ & $u^{2}+u v$ \\
\hline 0 & 0 & $-u^{2} v+v^{3}$ & $-u^{2} v$ & $-u^{2} v-u v^{2}$ \\
$u^{2}-v^{2}$ & $u^{3}-u v^{2}$ & $u^{3}-u^{2} v-u v^{2}+v^{3}$ & $u^{3}-u^{2} v-u v^{2}$ & $u^{3}-u^{2} v-2 u v^{2}$ \\
$u^{2}$ & $u^{3}$ & $u^{3}-u^{2} v+v^{3}$ & $u^{3}-u^{2} v$ & $u^{3}-u^{2} v-u v^{2}$ \\
$u^{2}+u v$ & $u^{3}+u^{2} v$ & $u^{3}+v^{3}$ & $u^{3}$ & $u^{3}-u v^{2}$ \\
\hline
\end{tabular}

The numbers $u^{3}, u^{3}-u v^{2}$, and $u^{3}-u^{2} v-u v^{2}$ occur twice in the table, and so $|g(A)| \leq 13$. The numbers in the table are decreasing from left to right in each row and increasing from top to bottom in each column. The following nine numbers in $g(A)$ form a strictly increasing sequence: 


$$
\begin{aligned}
-u^{2} v-u v^{2} & <-u^{2} v<-u^{2} v+v^{3}<0<u^{3}-u^{2} v \\
& <u^{3}-u^{2} v+v^{3}<u^{3}<u^{3}+v^{3}<u^{3}+3 u^{2} v .
\end{aligned}
$$

The other four numbers satisfy

$$
u^{3}-u^{2} v-2 u v^{2}<u^{3}-u^{2} v-u v^{2}<u^{3}-u^{2} v-u v^{2}+v^{3}<u^{3}-u v^{2} .
$$

Indeed, there is no pair of expressions in the table whose difference has last coefficient greater than 2 , and so $|g(A)|=13$.

Finally, we consider the sets $f(B)$ and $g(B)$ :

\begin{tabular}{ccccc}
\hline$f(B)$ & 0 & $u^{2}-u v$ & $u^{2}-v^{2}$ & $u^{2}$ \\
\hline 0 & 0 & $u^{2} v-u v^{2}$ & $u^{2} v-v^{3}$ & $u^{2} v$ \\
$u^{2}-u v$ & $u^{3}-u^{2} v$ & $u^{3}-u v^{2}$ & $u^{3}-v^{3}$ & $u^{3}$ \\
$u^{2}-v^{2}$ & $u^{3}-u v^{2}$ & $u^{3}+u^{2} v-2 u v^{2}$ & $u^{3}+u^{2} v-u v^{2}-v^{3}$ & $u^{3}+u^{2} v-u v^{2}$ \\
\cline { 2 - 5 }$u^{2}$ & $u^{3}$ & $u^{3}+u^{2} v-u v^{2}$ & $u^{3}+u^{2} v-v^{3}$ & $u^{3}+u^{2} v$ \\
\hline$g(B)$ & 0 & $u^{2}-u v$ & $u^{2}-v^{2}$ & $u^{2}$ \\
\hline 0 & 0 & $-u^{2} v+u v^{2}$ & $-u^{2} v+v^{3}$ & $-u^{2} v$ \\
$u^{2}-u v$ & $u^{3}-u^{2} v$ & $u^{3}-2 u^{2} v+u v^{2}$ & $u^{3}-2 u^{2} v+v^{3}$ & $u^{3}-2 u^{2} v$ \\
$u^{2}-v^{2}$ & $u^{3}-u v^{2}$ & $u^{3}-u^{2} v$ & $u^{3}-u^{2} v-u v^{2}+v^{3}$ & $u^{3}-u^{2} v-u v^{2}$ \\
$u^{2}$ & $u^{3}$ & $u^{3}-u^{2} v+u v^{2}$ & $u^{3}-u^{2} v+v^{3}$ & $u^{3}-u^{2} v$ \\
\hline
\end{tabular}

In the table for $f(B)$, the numbers $u^{3}, u^{3}-u v^{2}$, and $u^{3}+u^{2} v-u v^{2}$ occur twice, and so $|f(B)| \leq 13$. In the table for $g(B)$, the number $u^{3}-u^{2} v$ occurs three times, so $|g(B)| \leq 14$. In neither table is there a pair of numbers whose difference has last coefficient greater than 2 , and so $|f(B)|=13$ and $|g(B)|=14$. This completes the proof.

THEOREM 5. Let $u$ and $v$ be relatively prime positive integers with $u>v$, and consider the forms

$$
f=u x+v y \quad \text { and } \quad g=u x-v y .
$$

If $A$ is an arithmetic progression of length $t \leq u$, then $|f(A)|=|g(A)|=t^{2}$.

Proof. Since an arithmetic progression of length $t$ is affinely equivalent to the interval $[0, t-1]$, it suffices to consider the sets $A_{t}=[0, t-1]$ for $t=1, \ldots, u$.

If $x_{1}, x_{2}, y_{1}, y_{2} \in A_{t}$ and $u x_{1}+v y_{1}=u x_{2}+v y_{2}$, then we have $u\left(x_{1}-x_{2}\right)$ $=v\left(y_{2}-y_{1}\right)$. Since $(u, v)=1$, it follows that $u$ divides $y_{2}-y_{1}$. Since $\left|y_{2}-y_{1}\right|$ $<t \leq u$, it follows that $y_{1}=y_{2}$, and so $x_{1}=x_{2}$. Thus, every element in $f\left(A_{t}\right)$ has a unique representation in the form $u x+v y$, and $\left|f\left(A_{t}\right)\right|=t^{2}$. The proof that $\left|g\left(A_{t}\right)\right|=t^{2}$ is similar. 


\section{The pair of linear forms $u x+v y$ and $x-y$}

THEOREM 6. Let $u$ and $v$ be relatively prime positive integers with $u>v$, and consider the linear forms

$$
f(x, y)=u x+v y, \quad d(x, y)=x-y .
$$

Let

$$
A=\left\{0, v^{3}, v^{3}+v^{2} u, v^{3}+v^{2} u+v u^{2}, v^{3}+v^{2} u+v u^{2}+u^{3}\right\} .
$$

Then

$$
|f(A)| \leq 19, \quad|d(A)|=21 .
$$

Proof. Let

$$
\begin{aligned}
& a_{0}=0, \\
& a_{1}=v^{3}, \\
& a_{2}=v^{3}+v^{2} u, \\
& a_{3}=v^{3}+v^{2} u+v u^{2}, \\
& a_{4}=v^{3}+v^{2} u+v u^{2}+u^{3} .
\end{aligned}
$$

Then $A=\left\{a_{0}, a_{1}, a_{2}, a_{3}, a_{4}\right\}$, and

$$
a_{0}<a_{1}<a_{2}<a_{3}<a_{4} .
$$

Since $|A|=5$, we have $|f(A)| \leq 25$ and $|d(A)| \leq 21$. To show that $|f(A)|$ $\leq 19$, it suffices to give six different integers, each of which has two distinct representations in $f(A)$. Here they are:

$$
\begin{aligned}
& n_{1}=u a_{1}+v a_{1}=u a_{0}+v a_{2}, \\
& n_{2}=u a_{2}+v a_{1}=u a_{0}+v a_{3}, \\
& n_{3}=u a_{2}+v a_{2}=u a_{1}+v a_{3}, \\
& n_{4}=u a_{3}+v a_{1}=u a_{0}+v a_{4}, \\
& n_{5}=u a_{3}+v a_{2}=u a_{1}+v a_{4}, \\
& n_{6}=u a_{3}+v a_{3}=u a_{2}+v a_{4} .
\end{aligned}
$$

A straightforward calculation shows that

$$
n_{1}<n_{2}<n_{3}<n_{4}<n_{5}<n_{6}
$$

and so $|f(A)| \leq 19$.

Next we prove that $|d(A)|=21$. Let $D=\left\{a_{j}-a_{i}: 0 \leq i<j \leq 4\right\}$. It suffices to prove that $|D|=10$. If $v=1$, then $A=\left\{1,1+u, 1+u+u^{2}\right.$, $\left.1+u+u^{2}+u^{3}\right\}$ is a Sidon set and $|D|=10$.

Suppose that $v \geq 2$. Since $u>v$ and $a_{0}=0$, we have

$$
a_{1}<a_{2}-a_{1}<a_{3}-a_{2}<a_{4}-a_{3}<a_{4}-a_{2}<a_{4}-a_{1}<a_{4}
$$


and

$$
a_{2}<a_{3}-a_{1}<a_{3}
$$

Let

$$
\begin{aligned}
& D_{1}=\left\{a_{1}, a_{2}-a_{1}, a_{3}-a_{2}, a_{4}-a_{3}, a_{4}-a_{2}, a_{4}-a_{1}, a_{4}\right\}, \\
& D_{2}=\left\{a_{2}, a_{3}-a_{1}, a_{3}\right\} .
\end{aligned}
$$

We must show that $D_{1} \cap D_{2}=\emptyset$. There are three cases.

CASE OF $a_{2}$. Since

$$
a_{2}-a_{1}<a_{2}<a_{4}-a_{2}
$$

it follows that if $a_{2} \in D_{1}$, then $a_{2}=a_{3}-a_{2}$ or $a_{2}=a_{4}-a_{3}$. If $a_{2}=a_{3}-a_{2}$, then $v^{2}(v+u)=v^{3}+v^{2} u=v u^{2}$, and $v(v+u)=u^{2}$. Since $(u, v)=1$, it follows that $v=1$. If $a_{2}=a_{4}-a_{3}$, then $v^{2}(v+u)=v^{3}+v^{2} u=u^{3}$ and $v=1$.

CASE OF $a_{3}-a_{1}$. Since

$$
a_{3}-a_{2}<a_{3}-a_{1}<a_{4}-a_{2},
$$

it follows that if $a_{3}-a_{1} \in D_{1}$, then $a_{3}-a_{1}=a_{4}-a_{3}$ and so $v(v+u)=u^{2}$. This implies that $v=1$.

CASE OF $a_{3}$. Since

$$
a_{3}-a_{2}<a_{3}<a_{4}-a_{1}
$$

it follows that if $a_{3} \in D_{1}$, then $a_{3}=a_{4}-a_{3}$ or $a_{3}=a_{4}-a_{2}$. If $a_{3}=a_{4}-a_{3}$, then $v\left(v^{2}+v u+u^{2}\right)=v^{3}+v^{2} u+v u^{2}=u^{3}$, and $v=1$. If $a_{3}=a_{4}-a_{2}$, then $v^{3}+v^{2} u+v u^{2}=v u^{2}+u^{3}$, hence $v^{2}(v+u)=u^{3}$ and $v=1$. This completes the proof.

4. The pair of linear forms $u x+v y$ and $x+y$. In Section 2 we solved Problem 4 for pairs of normalized binary linear forms $f(x, y)=u_{1} x+v_{1} y$ and $g(x, y)=u_{2} x+v_{2} y$ with $u_{1}, u_{2} \geq 2$. In Section 3 we solved the case $f(x, y)=u x+v y$ with $u \geq 2$ and $d(x, y)=x-y$. The remaining case is $f(x, y)=u x+v y$ with $u \geq 2$ and $s(x, y)=x+y$.

For example, consider the form $f(x, y)=2 x+y$. We have

$$
4=|f(\{0,1\})|>|s(\{0,1\})|=3 .
$$

We shall construct a set $A$ with $|f(A)|<|s(A)|$. Start by defining the four sets

$$
\begin{aligned}
& R_{13}=\{0,1,6,7,9,11\}, \\
& R_{15}=\{0,1,5,6,10,11,13\}, \\
& R_{16}=\{0,1,3,5,7,9,11,13,15\}, \\
& R_{19}=\{0,1,11,12,14,16,18\} .
\end{aligned}
$$


Note that

$$
13 \cdot 15 \cdot 16 \cdot 19=59280
$$

and

$$
\left|R_{13}\right| \cdot\left|R_{15}\right| \cdot\left|R_{16}\right| \cdot\left|R_{19}\right|=6 \cdot 7 \cdot 9 \cdot 7=2646 .
$$

Let $x \bmod m$ denote the least nonnegative integer that is congruent to $x$ modulo $m$. We define

$$
A=\left\{x \in[1,59280]: x \bmod m \in R_{m} \text { for all } m \in\{13,15,16,19\}\right\} .
$$

The set $A$ contains 2646 elements. By direct calculation, we have $|f(A)|=$ 108014 and $|s(A)|=114575$.

The linear form $f(x, y)=2 x+y$ is a special case. In general, we do not have an algorithm to construct finite sets $A$ of integers such that $|f(A)|<$ $|s(A)|$ for an arbitrary normalized bilinear form $f(x, y)=u x+v y$ with $u \geq 2$. However, such sets do exist. In the following sections we shall show that, associated to the form $f(x, y)=u x+v y$, there is an infinite set $M$ of positive integers with the property that, for each $m \in M$, there is a set of congruence classes $R_{m} \subseteq \mathbb{Z} / m \mathbb{Z}$ such that $s\left(R_{m}\right)=\mathbb{Z} / m \mathbb{Z}$ and $f\left(R_{m}\right)$ $\subsetneq \mathbb{Z} / m \mathbb{Z}$. From the sets $R_{m}$ we construct a finite set $A$ of nonnegative integers such that $|f(A)|<|s(A)|$. Thus, we combine local solutions of the inequality to construct a global solution.

\section{A local to global criterion for pairs of linear forms in $n$ vari- ables}

Lemma 2. Let $f\left(x_{1}, \ldots, x_{n}\right)$ be a polynomial with integer coefficients. Let $m_{1}, \ldots, m_{r}$ be pairwise relatively prime positive integers, and $m=m_{1} \cdots m_{r}$. Let $R_{m_{i}}$ be a set of congruence classes modulo $m_{i}$ for $i=1, \ldots, r$. Let $R_{m}$ be the set of all congruence classes $a+m \mathbb{Z}$ such that $a+m_{i} \mathbb{Z} \in R_{m_{i}}$ for $i=1, \ldots, r$. Then

$$
\left|R_{m}\right|=\prod_{i=1}^{r}\left|R_{m_{i}}\right| \quad \text { and } \quad\left|f\left(R_{m}\right)\right|=\prod_{i=1}^{r}\left|f\left(R_{m_{i}}\right)\right| .
$$

Proof. This follows from the Chinese remainder theorem.

Lemma 3. Let $f\left(x_{1}, \ldots, x_{n}\right)=u_{1} x_{1}+\cdots+u_{n} x_{n}$ be a linear form with integer coefficients, and let

$$
h_{f}=\left|u_{1}\right|+\cdots+\left|u_{n}\right| .
$$

Let $R_{m}$ be a set of congruence classes in $\mathbb{Z} / m \mathbb{Z}$, and let $A$ be the set of integers that consists of the least nonnegative element of each congruence class in $R_{m}$. Then

$$
\left|f\left(R_{m}\right)\right| \leq|f(A)| \leq 2 h_{f}\left|f\left(R_{m}\right)\right|
$$


Proof. The triangle inequality implies that

$$
\left|f\left(a_{1}, \ldots, a_{n}\right)\right| \leq h_{f} \max \left(\left|a_{i}\right|: i=1, \ldots, n\right)
$$

for all integers $a_{1}, \ldots, a_{n}$. Since $A \subseteq[0, m-1]$, it follows that $f(A) \subseteq$ $\left[-h_{f}(m-1), h_{f}(m-1)\right]$. If $a \in f(A)$, then $a+m \mathbb{Z} \in f\left(R_{m}\right)$. The lower bound in (2) follows from the fact that $f(A)$ contains at least one element of every congruence class in $f\left(R_{m}\right)$. The upper bound in (2) follows from the fact that the interval $\left[-h_{f}(m-1), h_{f}(m-1)\right]$ contains at most $2 h_{f}$ members of any congruence class modulo $m$.

TheOREM 7. Let $f\left(x_{1}, \ldots, x_{n}\right)=u_{1} x_{1}+\cdots+u_{n} x_{n}$ and $g\left(x_{1}, \ldots, x_{n}\right)=$ $v_{1} x_{1}+\cdots+v_{n} x_{n}$ be binary linear forms. Let $M=\left\{m_{i}\right\}_{i=1}^{\infty}$ be a set of pairwise relatively prime integers such that $m_{i} \geq 2$ for all $m_{i} \in M$. If for every $m_{i} \in M$ there exists a nonempty set $R_{m_{i}}$ of congruence classes in $\mathbb{Z} / m_{i} \mathbb{Z}$ such that

$$
\prod_{i=1}^{\infty} \frac{\left|f\left(R_{m_{i}}\right)\right|}{\left|g\left(R_{m_{i}}\right)\right|}=0,
$$

then there is a finite set $A$ of integers such that

$$
|f(A)|<|g(A)| \text {. }
$$

Proof. Let

$$
h_{f}=\left|u_{1}\right|+\cdots+\left|u_{n}\right| .
$$

Since the infinite product (3) diverges to 0 , there is an integer $r$ such that

$$
\prod_{i=1}^{r} \frac{\left|f\left(R_{m_{i}}\right)\right|}{\left|g\left(R_{m_{i}}\right)\right|}<\frac{1}{2 h_{f}} .
$$

Let $m=m_{1} \cdots m_{r}$ and let $R_{m}$ be the set of all congruence classes $a+m \mathbb{Z}$ such that $a+m_{i} \mathbb{Z} \in R_{m_{i}}$ for $i=1, \ldots, r$. By Lemma 2 ,

$$
\frac{\left|f\left(R_{m}\right)\right|}{\left|g\left(R_{m}\right)\right|}=\prod_{i=1}^{r} \frac{\left|f\left(R_{m_{i}}\right)\right|}{\left|g\left(R_{m_{i}}\right)\right|} .
$$

Let $A$ be the set of integers that consists of the least nonnegative element in each congruence class in $R_{m}$. By Lemma 3 we have

$$
|f(A)| \leq 2 h_{f}\left|f\left(R_{m}\right)\right|<\left|g\left(R_{m}\right)\right| \leq|g(A)| .
$$

This completes the proof.

THEOREM 8. Let $f\left(x_{1}, \ldots, x_{n}\right)=u_{1} x_{1}+\cdots+u_{n} x_{n}$ and $g\left(x_{1}, \ldots, x_{n}\right)=$ $v_{1} x_{1}+\cdots+v_{n} x_{n}$ be binary linear forms. Let $M=\left\{m_{i}\right\}_{i=1}^{\infty}$ be a set of pairwise relatively prime positive integers such that $m_{i} \geq 2$ for all $m_{i} \in M$ and

$$
\sum_{i=1}^{\infty} \frac{1}{m_{i}}=\infty \text {. }
$$


If for every $m_{i} \in M$ there exists a nonempty set $R_{m_{i}}$ of congruence classes in $\mathbb{Z} / m_{i} \mathbb{Z}$ such that

$$
f\left(R_{m_{i}}\right) \neq \mathbb{Z} / m_{i} \mathbb{Z} \quad \text { and } \quad g\left(R_{m_{i}}\right)=\mathbb{Z} / m_{i} \mathbb{Z}
$$

then there is a finite set $A$ of integers such that

$$
|f(A)|<|g(A)| \text {. }
$$

Proof. Since $\left|f\left(R_{m_{i}}\right)\right| \leq m_{i}-1$ and $\left|g\left(R_{m_{i}}\right)\right|=m_{i}$ for all $m_{i} \in M$, we have

$$
\frac{\left|f\left(R_{m_{i}}\right)\right|}{\left|g\left(R_{m_{i}}\right)\right|} \leq 1-\frac{1}{m_{i}}
$$

The divergence of the infinite series $\sum_{i=1}^{\infty} m_{i}^{-1}$ implies that

$$
\prod_{i=1}^{\infty} \frac{\left|f\left(R_{m_{i}}\right)\right|}{\left|g\left(R_{m_{i}}\right)\right|}=\prod_{i=1}^{\infty}\left(1-\frac{1}{m_{i}}\right)=0,
$$

and the result follows immediately from Theorem 7 .

We can restate Theorem 8 as follows.

TheOREM 9. Let $f\left(x_{1}, \ldots, x_{n}\right)=u_{1} x_{1}+\cdots+u_{n} x_{n}$ and $g\left(x_{1}, \ldots, x_{n}\right)=$ $v_{1} x_{1}+\cdots+v_{n} x_{n}$ be binary linear forms. Let $M=\left\{m_{i}\right\}_{i=1}^{\infty}$ be a set of pairwise relatively prime positive integers such that $m_{i} \geq 2$ for all $m_{i} \in M$ and

$$
\sum_{i=1}^{\infty} \frac{1}{m_{i}}=\infty
$$

If for every $m_{i} \in M$ there exists an integer $q_{m_{i}}$ and a finite set $A_{m_{i}}$ of integers such that

(i) $f\left(a_{1}, \ldots, a_{n}\right) \not \equiv q_{m_{i}}\left(\bmod m_{i}\right)$ for all $a_{1}, \ldots, a_{n} \in A_{m_{i}}$, and

(ii) for every integer $q$ the congruence $g\left(a_{1}, \ldots, a_{n}\right) \equiv q(\bmod m)$ is solvable with $a_{1}, \ldots, a_{n} \in A_{m_{i}}$,

then there is a finite set $A$ of integers such that

$$
|f(A)|<|g(A)| \text {. }
$$

\section{An application of quadratic reciprocity}

TheOREM 10. Let $p$ be a prime number such that $p \equiv 1(\bmod 4)$ and $p>5$, and let

$$
R_{p}=\left\{k^{2}+p \mathbb{Z}: k=1, \ldots,(p-1) / 2\right\}
$$

be the set of quadratic residues modulo $p$. Let $s(x, y)=x+y$ and $d(x, y)=$ $x-y$. Then

$$
s\left(R_{p}\right)=d\left(R_{p}\right)=\mathbb{Z} / p \mathbb{Z}
$$


Proof. Since $p \equiv 1(\bmod 4)$ there is an integer $s \not \equiv 0(\bmod p)$ with $s^{2} \equiv-1$ $(\bmod p)$. For all integers $a$ and $u$ with $u \not \equiv 0(\bmod p)$ there is an integer $v$ (unique modulo $p)$ such that $u v \equiv a(\bmod p)$. Let $x \equiv(u-v) / 2(\bmod p)$ and $y \equiv(u+v) / 2(\bmod p)$. Then $y^{2}+(s x)^{2} \equiv y^{2}-x^{2} \equiv a(\bmod p)$, and this congruence has at least $p-1$ solutions. Since there are at most two solutions with $x \equiv 0(\bmod p)$ and at most two solutions with $y \equiv 0(\bmod p)$, it follows that if $p>5$ then there is at least one solution with $x^{2}+p \mathbb{Z} \in R_{p}$ and $y^{2}+p \mathbb{Z} \in R_{p}$. Therefore, $s\left(R_{p}\right)=d\left(R_{p}\right)=\mathbb{Z} / p \mathbb{Z}$.

TheOREM 11. Consider the binary linear form

$$
f(x, y)=u x+v y
$$

where $u$ and $v$ are integers not divisible by $p$. Let $p$ be a prime number and let $R_{p}$ be the set of quadratic residues modulo $p$. Then $p \mathbb{Z} \in f\left(R_{p}\right)$ if and only if $-u v$ is a quadratic residue modulo $p$.

Proof. If $p \mathbb{Z} \in f\left(R_{p}\right)$, then there are integers $k_{1}$ and $k_{2}$ not divisible by $p$ such that

$$
u k_{1}^{2}+v k_{2}^{2} \equiv 0(\bmod p) .
$$

Then

$$
u v k_{1}^{2}+\left(v k_{2}\right)^{2} \equiv 0(\bmod p)
$$

and so

$$
-u v \equiv\left(v k_{2} k_{1}^{-1}\right)^{2}(\bmod p),
$$

that is, $-u v$ is a quadratic residue modulo $p$.

Conversely, if $-u v$ is a quadratic residue modulo $p$, then there is an integer $z \not \equiv 0(\bmod p)$ such that $-u v \equiv z^{2}(\bmod p)$ and so

$$
f\left(v^{2}, z^{2}\right)=u v^{2}+v z^{2} \equiv 0(\bmod p) .
$$

Thus, $p \mathbb{Z}=f\left(v^{2}+p \mathbb{Z}, z^{2}+p \mathbb{Z}\right) \in f\left(R_{p}\right)$.

THEOREM 12. Let

$$
f(x, y)=u x+v y
$$

be a normalized bilinear form such that $|u v|$ is not a perfect square. Let

$$
s(x, y)=x+y \quad \text { and } \quad d(x, y)=x-y .
$$

There exist finite sets $A$ and $A^{\prime}$ of integers such that

$$
|f(A)|<|s(A)|, \quad\left|f\left(A^{\prime}\right)\right|<\left|d\left(A^{\prime}\right)\right| .
$$

Proof. Since $f(x, y)$ is normalized and $|u v|$ is not a square, we can write

$$
|u v|=w^{2} 2^{\varepsilon} \prod_{j=1}^{t} q_{j}
$$


where $w$ is a positive integer, $\varepsilon \in\{0,1\}, q_{1}, \ldots, q_{t}$ are distinct odd primes, and $q_{i} \neq p$ for $i=1, \ldots, p$. For $p \equiv 1(\bmod 4)$, we have

$$
\left(\frac{-u v}{p}\right)=\left(\frac{ \pm w^{2} 2^{\varepsilon} \prod_{j=1}^{t} q_{j}}{p}\right)=\left(\frac{2}{p}\right)^{\varepsilon} \prod_{j=1}^{t}\left(\frac{q_{j}}{p}\right)=\left(\frac{2}{p}\right)^{\varepsilon} \prod_{j=1}^{t}\left(\frac{p}{q_{j}}\right) .
$$

If $\varepsilon=1$, we choose $p$ so that $p \equiv 5(\bmod 8)$ and $p \equiv 1\left(\bmod q_{j}\right)$ for $j=1, \ldots, t$. Then $(-u v \mid p)=-1$. If $\varepsilon=0$, then $t \geq 1$ and we choose $p$ so that $p \equiv 1(\bmod 4), p \equiv 1\left(\bmod q_{j}\right)$ for $j=2, \ldots, t$, and $\left(p \mid q_{1}\right)=-1$. Again, $(-u v \mid p)=-1$. In both cases, there is at least one infinite arithmetic progression $P(u, v)$ such that if $p$ is a prime and $p \in P(u, v)$, then $p \equiv 1(\bmod 4)$ and $(-u v \mid p)=-1$. By Dirichlet's theorem, the arithmetic progression $P(u, v)$ contains infinitely many primes and

$$
\sum_{\substack{p \in P(u, v) \\ p \text { prime }}} \frac{1}{p}=\infty
$$

By Theorems 10 and 11 , for each prime $p \in P(u, v)$, the set

$$
R_{p}=\left\{k^{2}+p \mathbb{Z}: k=1, \ldots,(p-1) / 2\right\}
$$

satisfies

$$
f\left(R_{p}\right) \neq \mathbb{Z} / p \mathbb{Z} \quad \text { and } \quad s\left(R_{p}\right)=d\left(R_{p}\right)=\mathbb{Z} / p \mathbb{Z} .
$$

The result now follows from Theorem 8 .

7. An exponential sum. After Theorem 12, we are left to consider only normalized bilinear forms $f(x, y)=u x+v y$ such that $|u v|$ is a square. Since $u$ and $v$ are relatively prime, it follows that there are positive integers $U$ and $V$ not divisible by $p$ such that $u=U^{2}$ and $v= \pm V^{2}$. Let $R_{p}$ denote the set of quadratic residues modulo the prime $p$. If $f(x, y)=U^{2} x+V^{2} y$, then $f\left(R_{p}\right)=s\left(R_{p}\right)$. If $f(x, y)=U^{2} x-V^{2} y$, then $f\left(R_{p}\right)=d\left(R_{p}\right)$. This suggests that considering only squares mod $p$ will not suffice to resolve Problem 4 in this remaining case. We shall generalize our method to $k$ th powers. We begin by applying elementary harmonic analysis on finite fields to binary linear forms. A general reference is Nathanson [5, Chapter 4].

Let $p$ be a prime number and $\mathbb{F}_{p}=\mathbb{Z} / p \mathbb{Z}$ the field of congruence classes modulo $p$. We denote the multiplicative group of the field by $\mathbb{F}_{p}^{\times}$and define

$$
e_{p}(t)=e^{2 \pi i t / p}
$$

For all integers $a, b$, and $t$ we have

$$
\sum_{t=0}^{p-1} e_{p}((a-b) t)= \begin{cases}0 & \text { if } a \neq \equiv b(\bmod p) \\ p & \text { if } a \equiv b(\bmod p)\end{cases}
$$


If $x$ is a congruence class modulo $p$, that is, if $x=t+p \mathbb{Z}$ for some integer $t$, then we define $e_{p}(x)=e_{p}(t)$. This function is well-defined on $\mathbb{F}_{p}$.

Let $\gamma$ be a complex-valued function on $\mathbb{F}_{p}$. We define the Fourier transform $\widehat{\gamma}: \mathbb{F}_{p} \rightarrow \mathbb{C}$ by

$$
\widehat{\gamma}(x)=\sum_{y \in \mathbb{F}_{p}} \gamma(y) e_{p}(-x y)
$$

We have

$$
\widehat{\gamma}(0)=\sum_{y \in \mathbb{F}_{p}} \gamma(y)
$$

and Plancherel's formula [5, Theorem 4.9]

$$
\sum_{x \in \mathbb{F}_{p}}|\widehat{\gamma}(x)|^{2}=p \sum_{x \in \mathbb{F}_{p}}|\gamma(x)|^{2}
$$

Let $H$ be a subset of $\mathbb{F}_{p}$ of cardinality $n$. We also use $H$ to denote the characteristic function of $H$, that is, $H: \mathbb{F}_{p} \rightarrow \mathbb{C}$ is the function defined by

$$
H(x)= \begin{cases}1 & \text { if } x \in H \\ 0 & \text { if } x \notin H\end{cases}
$$

Then

$$
\widehat{H}(x)=\sum_{y \in \mathbb{F}_{p}} H(y) e_{p}(-x y)=\sum_{h \in H} e_{p}(-x h), \quad \widehat{H}(0)=\operatorname{card}(H)=n .
$$

Applying Plancherel's formula to the function $H$, we obtain

$$
\sum_{x \in \mathbb{F}_{p}}|\widehat{H}(x)|^{2}=p \sum_{x \in \mathbb{F}_{p}}|H(x)|^{2}=p \operatorname{card}(H)=p n
$$

and so

$$
\sum_{x \in \mathbb{F}_{p}^{\times}}|\widehat{H}(x)|^{2}=\sum_{x \in \mathbb{F}_{p}}|\widehat{H}(x)|^{2}-|\widehat{H}(0)|^{2}=(p-n) n .
$$

THEOREM 13. Let $f(x, y)=u x+v y$, where $u$ and $v$ are integers not divisible by $p$. Let $H$ be a subgroup of order $n \geq 2$ of the multiplicative group $\mathbb{F}_{p}^{\times}$and let

$$
k=\left[\mathbb{F}_{p}^{\times}: H\right]=\frac{p-1}{n} .
$$

If $p>k^{4}$, then $\mathbb{F}_{p}^{\times} \subseteq f(H)$, that is, every element of $\mathbb{F}_{p}^{\times}$can be represented in the form $f\left(h_{1}, h_{2}\right)$ for some $h_{1}, h_{2} \in H$.

Proof. Define the representation function $r: \mathbb{F}_{p} \rightarrow \mathbb{N}_{0}$ as follows. For every $x \in \mathbb{F}_{p}$, let $r(x)$ denote the number of ordered pairs $\left(h_{1}, h_{2}\right) \in H \times H$ such that $f\left(h_{1}, h_{2}\right)=x$. Then

$$
\sum_{x \in \mathbb{F}_{p}} r(x)=|H|^{2}=n^{2}
$$


and

$$
\begin{aligned}
\widehat{r}(x) & =\sum_{y \in \mathbb{F}_{p}} r(y) e_{p}(-x y)=\sum_{y \in \mathbb{F}_{p}}\left(\sum_{\substack{h_{1}, h_{2} \in H \\
u h_{1}+v h_{2}=y}} 1\right) e_{p}(-x y) \\
& =\sum_{h_{1} \in H} \sum_{h_{2} \in H} e_{p}\left(-\left(u h_{1}+v h_{2}\right) x\right)=\sum_{h_{1} \in H} e_{p}\left(-u x h_{1}\right) \sum_{h_{2} \in H} e_{p}\left(-v x h_{2}\right) \\
& =\widehat{H}(u x) \widehat{H}(v x) .
\end{aligned}
$$

Applying Plancherel's formula (4) to the function $r(x)$, we obtain

$$
\begin{aligned}
\sum_{x \in \mathbb{F}_{p}^{\times}}|\widehat{H}(u x) \widehat{H}(v x)|^{2} & =\sum_{x \in \mathbb{F}_{p}}|\widehat{H}(u x) \widehat{H}(v x)|^{2}-|\widehat{H}(0)|^{4}=\sum_{x \in \mathbb{F}_{p}} \widehat{r}(x)^{2}-n^{4} \\
& =p \sum_{x \in \mathbb{F}_{p}} r(x)^{2}-n^{4}=p \sum_{x \in \mathbb{F}_{p}}\left(r(x)-\frac{n^{2}}{p}\right)^{2} .
\end{aligned}
$$

Let $x, x^{\prime} \in \mathbb{F}_{p}^{\times}$. If $x$ and $x^{\prime}$ belong to the same coset of $\mathbb{F}_{p} / H$, then there exists $h^{\prime} \in H$ such that $x=x^{\prime} h^{\prime}$. It follows that

$$
\widehat{H}(x)=\sum_{h \in H} e_{p}(-x h)=\sum_{h \in H} e_{p}\left(-x^{\prime} h^{\prime} h\right)=\sum_{h \in H} e_{p}\left(-x^{\prime} h\right)=\widehat{H}\left(x^{\prime}\right)
$$

and so the Fourier transform $\widehat{H}(x)$ is constant on the cosets of $\mathbb{F}_{p}^{\times} / H$. Similarly, $u h_{1}+v h_{2}=x^{\prime}$ if and only if $u h_{1} h^{\prime}+v h_{2} h^{\prime}=x^{\prime} h^{\prime}=x$, and so $r(x)=r\left(x^{\prime}\right)$, that is, the representation function $r(x)$ is also constant on the cosets of $\mathbb{F}_{p}^{\times} / H$.

Let $\left\{x_{1}, \ldots, x_{k}\right\} \subseteq \mathbb{F}_{p}^{\times}$be a set of coset representatives of $H$, that is,

$$
\mathbb{F}_{p}^{\times} / H=\left\{x_{1} H, \ldots, x_{k} H\right\} .
$$

Applying (5), we obtain

$$
(p-n) n=\sum_{x \in \mathbb{F}_{p}^{\times}}|\widehat{H}(x)|^{2}=\sum_{i=1}^{k} \sum_{x \in x_{i} H}|\widehat{H}(x)|^{2}=n \sum_{i=1}^{k}\left|\widehat{H}\left(x_{i}\right)\right|^{2}
$$

and so

$$
\sum_{i=1}^{k}\left|\widehat{H}\left(x_{i}\right)\right|^{2}=p-n
$$

For every $x \in \mathbb{F}_{p}^{\times}$, there is an integer $j \in\{1, \ldots, k\}$ such that $x \in x_{j} H$ and $\widehat{H}(x)=\widehat{H}\left(x_{j}\right)$. It follows that

$$
|\widehat{H}(x)|^{2}=\left|\widehat{H}\left(x_{j}\right)\right|^{2} \leq \sum_{i=1}^{k}\left|\widehat{H}\left(x_{i}\right)\right|^{2}=p-n .
$$


Since $(u, p)=(v, p)=1$, we have

$$
\begin{aligned}
p \sum_{x \in \mathbb{F}_{p}}\left(r(x)-\frac{n^{2}}{p}\right)^{2} & =\sum_{x \in \mathbb{F}_{p}^{\times}}|\widehat{H}(u x) \widehat{H}(v x)|^{2} \leq(p-n) \sum_{x \in \mathbb{F}_{p}^{\times}}|\widehat{H}(u x)|^{2} \\
& =(p-n) \sum_{x \in \mathbb{F}_{p}^{\times}}|\widehat{H}(x)|^{2}=(p-n)^{2} n .
\end{aligned}
$$

Since the representation function $r(x)$ is constant on cosets of $H$, we have

$$
\begin{aligned}
\sum_{i=1}^{k}\left(r\left(x_{i}\right)-\frac{n^{2}}{p}\right)^{2} & =\frac{1}{n} \sum_{i=1}^{k} \sum_{x \in x_{i} H}\left(r(x)-\frac{n^{2}}{p}\right)^{2}=\frac{1}{n} \sum_{x \in \mathbb{F}_{p}}\left(r(x)-\frac{n^{2}}{p}\right)^{2} \\
& \leq \frac{(p-n)^{2}}{p} .
\end{aligned}
$$

For every $x \in \mathbb{F}_{p}^{\times}$, we have $x \in x_{j} H$ for some $j$, and so

$$
\left(r(x)-\frac{n^{2}}{p}\right)^{2}=\left(r\left(x_{j}\right)-\frac{n^{2}}{p}\right)^{2} \leq \frac{(p-n)^{2}}{p} .
$$

If $r(x)=0$ for some $x \in \mathbb{F}_{p}^{\times}$, then

$$
\frac{n^{4}}{p^{2}} \leq \frac{(p-n)^{2}}{p}
$$

Since $|H|=n \geq 2$ and $p(p-n) \leq p(p-2)<(p-1)^{2}$, we have

$$
\left(\frac{p-1}{k}\right)^{4}=n^{4} \leq p(p-n)^{2}<(p-1)^{3} \quad \text { and } \quad p \leq k^{4} \text {. }
$$

This proves that if $p>k^{4}$, then $r(x) \geq 1$ for all $x \in \mathbb{F}_{p}^{\times}$and so $\mathbb{F}_{p}^{\times} \subseteq f(H)$.

A finite cyclic group $G$ of order $N$ has a unique subgroup $H$ of order $n$ for every positive divisor $n$ of $N$. If $k=[G: H]=N / n$ and if $g$ is a generator of $G$, then $H=\left\{g^{i k}: i=0,1, \ldots, n-1\right\}=\left\{x^{k}: x \in G\right\}$ is the set of $k$ th powers in $G$.

Let $H$ be a subgroup of order $n$ of $\mathbb{F}_{p}^{\times}$and let $k=\left[\mathbb{F}_{p}^{\times}: H\right]=(p-1) / n$. Since the multiplicative group of a finite field is cyclic, it follows that $H$ is the subgroup of $k$ th powers $\bmod p$, that is, $H=\left\{x^{k}: x \in \mathbb{F}_{p}^{\times}\right\}$. We can restate Theorem 13 as follows.

THEOREM 14. Let $f(x, y)=u x+v y$ be a binary linear form with nonzero integral coefficients $u$ and $v$. For $k \geq 2$, let $p$ be a prime number such that $p \equiv 1(\bmod k), p>k^{4}$, and $(p, u v)=1$. If $H$ is the set of $k$ th powers in $\mathbb{F}_{p}^{\times}$, then

$$
\mathbb{F}_{p}^{\times} \subseteq f(H)
$$


We shall prove that if $u \geq 2$, then there are infinitely many primes $p$ such that $0 \notin f(H)$ and so $f(H)=\mathbb{F}_{p}^{\times}$. We use a standard result about irreducible polynomials: If $a$ is a nonzero rational number and $q$ is a prime number such that $a$ is not a $q$ th power, then the polynomial $g(x)=x^{q}-a$ is irreducible in $\mathbb{Q}[x]$.

THEOREM 15. Let $u$ and $v$ be relatively prime integers such that $u>$ $|v| \geq 1$. Let

$$
f(x, y)=u x+v y
$$

There exist finite sets $A$ and $A^{\prime}$ of integers such that

$$
|f(A)|<|s(A)| \text { and }\left|f\left(A^{\prime}\right)\right|<\left|d\left(A^{\prime}\right)\right| .
$$

Proof. Let $k$ be a positive integer and let $p$ be a prime such that $p \equiv 1$ $(\bmod k)$. Let $H$ be the set of $k$ th powers of elements of $\mathbb{F}_{p}^{\times}$. Then $H$ is a multiplicative subgroup of $\mathbb{F}_{p}^{\times}$of order $|H|=(p-1) / k$ and $\left[\mathbb{F}_{p}^{\times}: H\right]=k$.

Let $u$ and $v$ be integers relatively prime to $p$, and consider the binary linear form

$$
f(x, y)=u x+v y .
$$

By Theorem 13, if $p>k^{4}$, then $\mathbb{F}_{p}^{\times} \subseteq f(H)$ and so

$$
|f(H)| \geq p-1 .
$$

Let $u=1$ and $v= \pm 1$. For the polynomial $d(x, y)=x-y$, we have

$$
d\left(1^{k}, 1^{k}\right)=0 \in d(H) \text { and so } d(H)=\mathbb{F}_{p} .
$$

If $k$ is odd, then for the polynomial $s(x, y)=x+y$ we have

$$
s\left(1^{k},(-1)^{k}\right)=0 \in s(H) \text { and } s(H)=\mathbb{F}_{p} .
$$

In both cases,

$$
|d(H)|=|s(H)|=p .
$$

Let $u>|v| \geq 1$ and $(u, v)=(u v, p)=1$. Let $a=-u v^{k-1}$. If there exist $h_{1}=\ell_{1}^{k} \in H$ and $h_{2}=\ell_{2}^{k} \in H$ such that

$$
f\left(h_{1}, h_{2}\right)=u h_{1}+v h_{2}=u \ell_{1}^{k}+v \ell_{2}^{k}=0,
$$

then

$$
\left(\frac{u \ell_{1}}{\ell_{2}}\right)^{k}+u^{k-1} v=0
$$

and the polynomial $g(x)=x^{k}-a$ has a root in $\mathbb{F}_{p}$, and is, therefore, reducible. It follows that if $g(x)$ is irreducible in $\mathbb{F}_{p}[x]$, then $0 \notin f(H)$ and so

$$
|f(H)|=p-1 .
$$

The rational integer $a=-u^{k-1} v$ is not a $q$ th power for all sufficiently large primes $q$, and so the polynomial $g(x)=x^{q}-a$ is irreducible over $\mathbb{Q}$. Let $\mathcal{P}$ be the set of primes $p>q^{4}$ such that $p \equiv 1(\bmod q)$ and $g(x)$ is 
irreducible in $\mathbb{F}_{p}[x]$. The Chebotarev density theorem implies that the series $\sum_{p \in \mathcal{P}} 1 / p$ diverges, and Theorem 15 now follows directly from Theorem 8 . This completes the proof.

\section{References}

[1] H. T. Croft, Research problems, Problem 7, Section 6, mimeographed notes, Univ. of Cambridge, 1967.

[2] P. Hegarty, Some explicit constructions of sets with more sums than differences, arXiv:math.NT/0611582, 2006.

[3] J. Marica, On a conjecture of Conway, Canad. Math. Bull. 12 (1969), 233-234.

[4] G. Martin and K. O'Bryant, Many sets have more sums than differences, arXiv: math.NT/0608131, 2006.

[5] M. B. Nathanson, Elementary Methods in Number Theory, Grad. Texts in Math. 195, Springer, New York, 2000.

[6] - Problems in additive number theory, I, arXiv:math.NT/0604340; to appear in Additive Combinatorics, CRM, Montreal, 2007.

[7] —, Sets with more sums than differences, Integers 7 (2007), A5.

Department of Mathematics

Lehman College (CUNY)

Bronx, NY 10468, U.S.A.

E-mail: melvyn.nathanson@lehman.cuny.edu

Department of Mathematics

College of Staten Island (CUNY)

Staten Island, NY 10314, U.S.A.

E-mail: kevin@member.ams.org

Department of Mathematics

CUNY Graduate Center

New York, NY 10036, U.S.A.

E-mail: borosz@gc.cuny.edu
Rényi Institute of Mathematics Hungarian Academy of Sciences Budapest, Hungary E-mail: ruzsa@renyi.hu

Department of Mathematics New University of Lisbon Lisbon, Portugal

E-mail: mnasilva@gmail.com 